\title{
AN ASTROMETRIC AND PHOTOMETRIC STUDY OF THE INTERMEDIATE-AGE OPEN CLUSTER NGC 2158 AND ITS ECLIPSING BINARY [NBN2015]78
}

\author{
A. E. Abdelaziz ${ }^{1}$, Y. H. M. Hendy ${ }^{1}$, A. Shokry ${ }^{1}$, S. M. Saad ${ }^{1,2}$, F. Y. Kamal ${ }^{3}$, and K. A. Edris ${ }^{3}$ \\ Received February 24 2020; accepted July 22020
}

\begin{abstract}
We present a photometric and astrometric analysis of the NGC 2158 cluster using Gaia DR2 and 2MASS data. The cluster age, color excess, intrinsic distance modulus and distance are calculated to be $t=2.240 \pm 0.260 \mathrm{Gyr}, E(B-V)=$ $0.420 \pm 0.050 \mathrm{mag},(m-M)_{\odot}=12.540 \pm 0.130 \mathrm{mag}$ and $d_{\odot}=3224 \pm 200 \mathrm{pc}$ respectively. The photometric analysis and light curve modelling of the proposed eclipsing binary member [NBN2015]78 is performed using the latest version of the WilsonDevinney (W-D) code. The solutions show that the system is an over-contact binary with a secondary component filling its Roche lobe, with a mass ratio $q=0.262$. The primary and the secondary components of the system consist of two late spectral types K1 and K2 respectively. The membership of [NBN2015]78 is discussed using two independent methods, and we find that [NBN2015]78 is an interloper and not a member of NGC 2158.
\end{abstract}

\section{RESUMEN}

Presentamos un análisis fotométrico y astrométrico del cúmulo NGC 2158 con datos de Gaia DR2 y 2MASS. Calculamos la edad del cúmulo, el exceso de color, el módulo intrínseco de distancia y la distancia como $t=2.240 \pm 0.260$ Gigaaños, $\quad E(B-V)=0.420 \pm 0.050 \mathrm{mag}, \quad(m-M)_{\odot}=12.540 \pm 0.130 \mathrm{mag} \quad \mathrm{y}$ $d_{\odot}=3224 \pm 200 \mathrm{pc}$, respectivamente. Mediante la última versión del código Wilson-Devinney modelamos la curva de luz de la binaria eclipsante [NBN2015]78 propuesta como miembro del cúmulo. Las soluciones muestran que el sistema es una binaria en sobre-contacto, cuya secundaria llena el lóbulo de Roche; el cociente de masas es $q=0.262$. Las dos componentes del sistema son de tipo espectral tardío, K1 y K2. Discutimos la pertenencia de [NBN2015]78 al cúmulo mediante dos métodos independientes, y encontramos que no pertenece a él.

Key Words: astrometry - binaries: eclipsing — open clusters and associations: individual: NGC 2158 - stars: individual: [NBN2015]78 - techniques: photometric

\section{INTRODUCTION}

Photometric studies of open clusters in the optical and infrared are fundamental tools for determining their structural parameters. These parameters play an important role towards understanding galactic structure and evolution. NGC 2158 [Melotte 40] is a rich northern open cluster of in-

\footnotetext{
${ }^{1}$ Astronomy Department, National Research Institute of Astronomy and Geophysics (NRIAG), Helwan, Cairo, Egypt.

${ }^{2}$ Kottamia Center of Scientificc Excellence for Astronomy and Space Sciences (KCScE), STDF No. 5217.

${ }^{3}$ Department of Astronomy and Meteorology, Faculty of Science, Al-Azhar University, Cairo, Egypt.
}

termediate age, located in the constellation Gemini (Carraro et al. 2002). It has a diameter of about $5^{\prime}$ (Lynga et al. 1987). Anderson et al. (2013) estimated a core radius $R_{c o r}=1^{\prime} .7$ and a limiting radius $R_{\text {lim }}=18^{\prime} .60$. Bedin et al. (2010) estimated $\log ($ age $)=9.301, E(B-V)=0.420 \mathrm{mag},(m-M)_{\odot}=$ $12.980 \mathrm{mag}$. Kharchenko et al. (2013) determined $\log ($ age $)=9.330, E(B-V)=0.333 \mathrm{mag}$ and a heliocentric distance of $d_{\odot}=4770 \mathrm{pc}$. NGC 2158 is centered at $(\mathrm{RA}=06: 07: 25.00, \mathrm{DE}=+24: 05: 48.00)$ and the eclipsing binary [NBN2015]78 is located at $(\mathrm{RA}=06: 07: 38.02, \mathrm{DE}=+24: 07: 24.94)$, Figure 1 dis- 


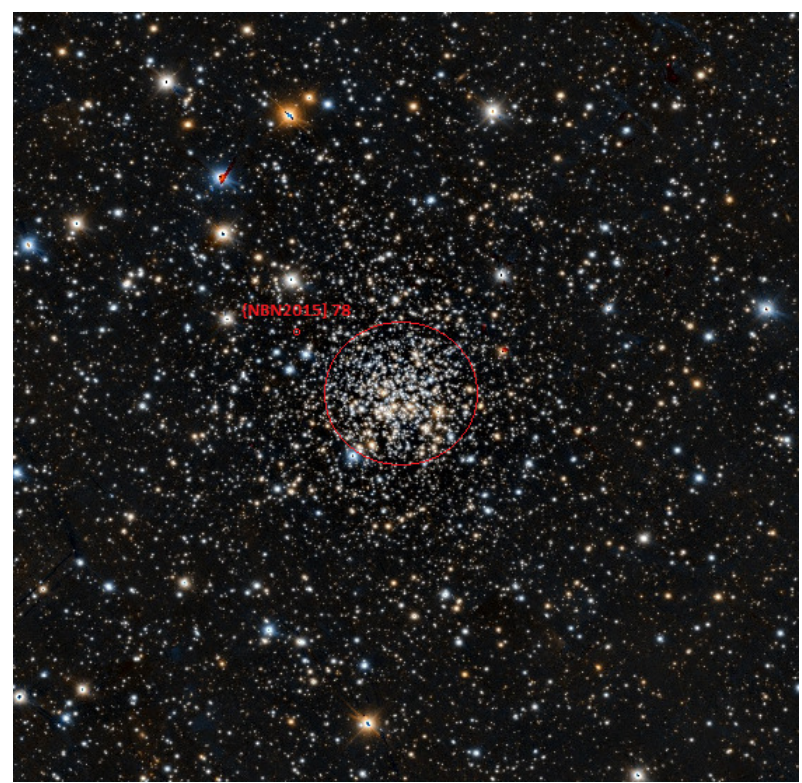

Fig. 1. Color image of the open cluster NGC 2158 from the Pan-STARRS survey (Panoramic Survey Telescope and Rapid Response System). The size of the field of view is $21^{\prime} \times 21^{\prime}$. The large red circle marks the region of the open cluster NGC 2158, while the small red circle gives the position of the eclipsing binary [NBN2015]78. The color figure can be viewed online.

plays the color image of the cluster using ALADIN (Bonnarel et al. 2000).

Studying eclipsing binary systems in the field and clusters gives valuable information about the physical and absolute parameters of the stars, such as masses and radii. Eclipsing binaries of the W UMa type are very important objects to study the evolutionary state of close binary stars and to characterize their formation. When they are members of a stellar cluster, the distance, age and metallicity of these stars are known independently, which makes them an ideal test for theories of binary structure and evolution. [NBN2015]78 is a poorly studied early-type eclipsing binary star which is located in the field of the open cluster NGC 2158. The designation comes from Nardiello et al. (2015), as it is the number 78 in their catalogue. It is also known as NGC 2158 MSS V60, NGC 2168 HIZ V6, and 2MASS J06073800 +2407249 . It is a short period (0.341325 days) eclipsing binary system (Nardiello et al. 2015).

The main aim of this work is to present the photometric and astrometric parameters for the open cluster NGC 2158 and to perform a light curve analysis and modelling of the eclipsing binary [NBN2015]78. This paper is organized as follows: the photometric and astrometric study of the open cluster NGC 2158 is presented in $\S 2$. The photometric study of the eclipsing binary [NBN2015]78 is presented in $\S 3$. Finally, discussions and conclusions are summarized in $\S 4$.

\section{PHOTOMETRIC AND ASTROMETRIC STUDY OF THE OPEN CLUSTER NGC 2158}

\subsection{Data and Analysis}

In the present work, we use both the GAIA DR2 and 2MASS databases. Astrometric, photometric and spectroscopic measurements of the entire sky have been obtained by the ESA mission Gaia (Gaia Collaboration et al. 2016). The latest version of the Gaia data (Gaia DR2) contains over 1.3 billion sources in three photometric bands $\left(G, G_{B P}, G_{R P}\right)$, in addition to their proper motion and parallax (Gaia Collaboration et al. 2018, Lindegren et al. 2018). The limiting $G$-band magnitude of Gaia DR2 data is $21 \mathrm{mag}$. At the bright end ( $G<14 \mathrm{mag})$, the uncertainties reach 0.02 mas in parallax and 0.05 mas/yr in proper motions, while for sources near $G \sim 21 \mathrm{mag}$ the uncertainties reach 2 mas and 5 mas/yr respectively. The 2MASS survey is the catalog of choice for astronomical studies near the Galactic plane (Skrutskie et al. 2006). The limiting magnitudes of 2MASS data $J(1.25 \mu \mathrm{m}), H(1.65 \mu \mathrm{m})$ and $K(2.17 \mu \mathrm{m})$ are $15.8,15.1$ and $14.3 \mathrm{mag}$, respectively.

We extracted the data around NGC 2158 inserting the coordinates of the cluster NGC 2158 as $\mathrm{RA}=06 \mathrm{~h} \quad 07 \mathrm{~m}$ 25s.00, $\mathrm{DEC}=+24^{\circ} 05^{\prime} \quad 48^{\prime \prime} \cdot 00$, $\ell=186.634$ and $b=+1.781$ (Dias et al. 2002) in the Virtual Observatory tool TOPCAT (Taylor 2005), within a radius of $30^{\prime}$. Cross-matching the Gaia DR2 and 2MASS data we obtain a matched number of stars of 17977.

To obtain the best astrometric precision of the proper motions, we used the sources brighter than $G=18 \mathrm{mag}$, which correspond to typical astrometric uncertainties smaller than 0.3 mas/yr in proper motion (Cantat-Gaudin et al. 2018, Lindegren et al. 2018). Using the Vector Point Diagram (VPD) of the proper motions in RA and DEC, we select the overdensity in the cluster region, obtaining 2103 stars (red region on the left panel, Figure 2). To obtain the candidate cluster members, we removed stars from the selected cluster region (red region) if their proper motions in RA and DEC were inconsistent with a median proper motion by more than three median absolute deviations (MAD). The color magnitude diagram (CMD) in the right panel of Figure 2 shows that the candidate members (red dots) fall on 

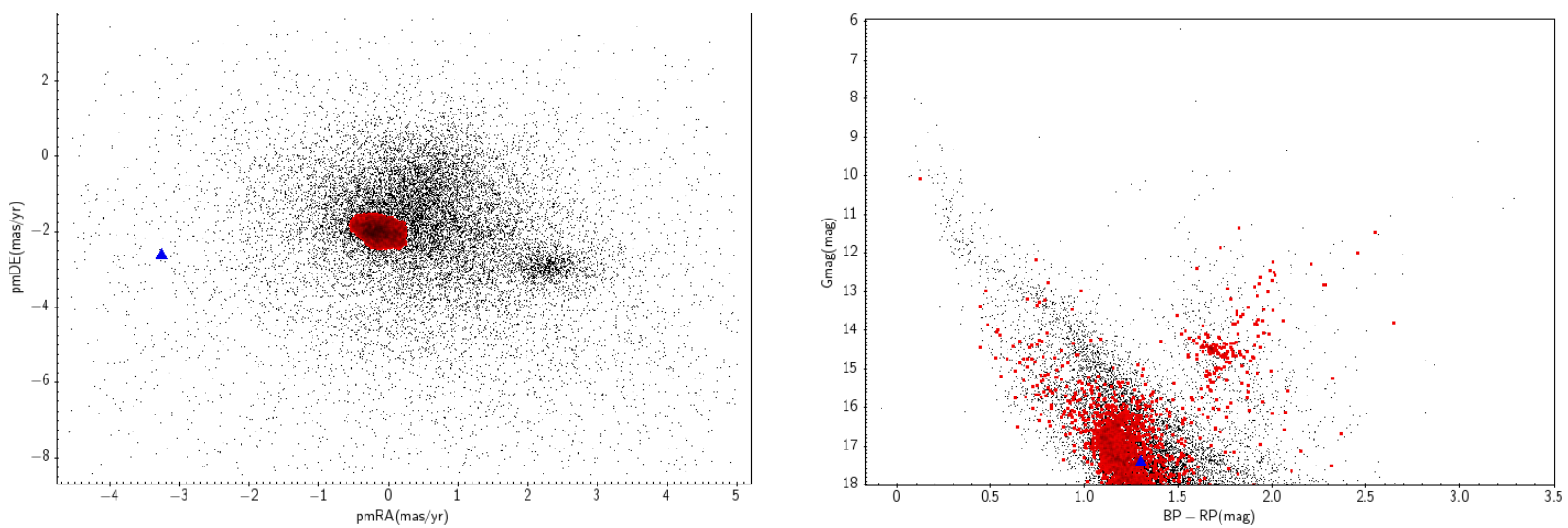

Fig. 2. The left panel shows the Vector Point Diagram (VPD), and the right panel shows the Color Magnitude Diagram (CMD) of the open cluster NGC 2158. The selected red region from the VPD marks the candidate member stars. The blue triangle and the black dots represent the eclipsing binary [NBN2015]78 and the field stars respectively. The red dots on the CMD represent candidate members. The color figure can be viewed online.
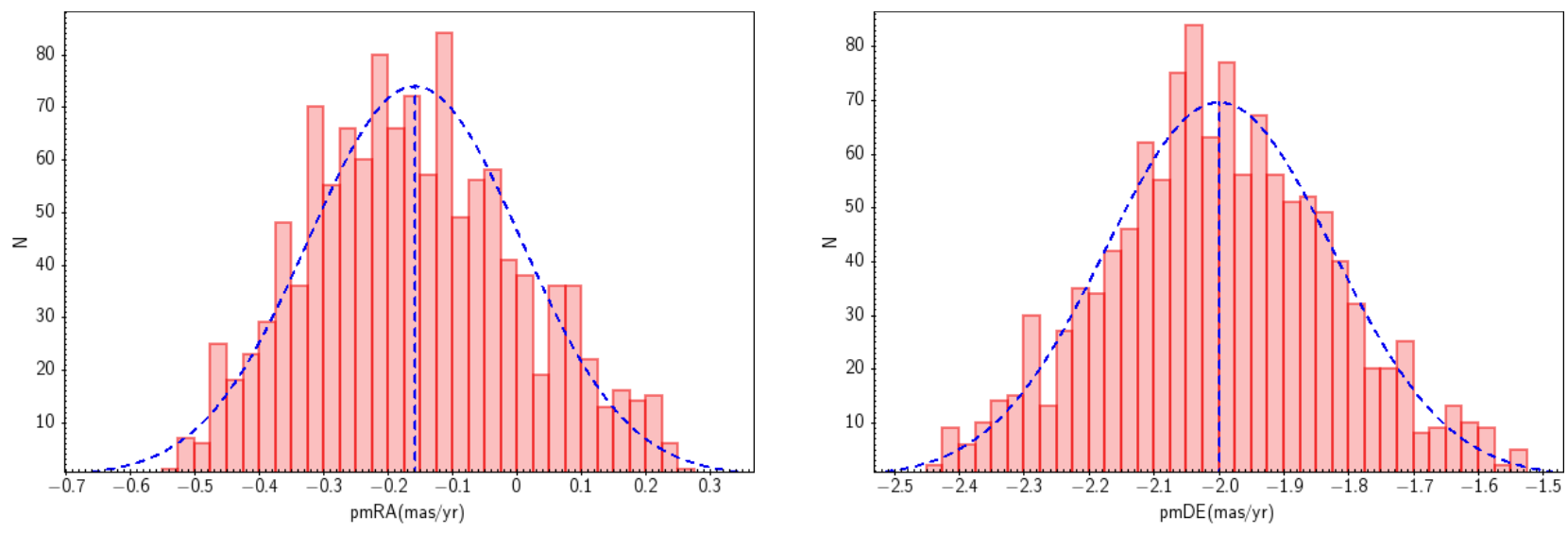

Fig. 3. The histograms of the proper motion in RA (mas/yr) and DEC (mas/yr), and the Gaussian fits are shown as blue dashed lines. The color figure can be viewed online.

a well-defined main sequence. The selected cluster region of the candidate members has a nearly ellipsoidal shape. The equivalent radius $R_{e}=\sqrt{a^{2}+b^{2}}$ (with $a$ and $b$ the semi-major and semi-minor axes of the ellipse, respectively) of the selected cluster region is $0.7 \mathrm{mas} / \mathrm{yr}$. This value is well within the range of the best-precision Gaia proper motions of 2 mas/yr (Ferreira et al. 2019).

Our membership criterion depends on the chosen overdensity region to define the members of the cluster from the VPD, which obviously is a compromise between losing cluster members and including field stars (see Bisht et al. 2019). To minimize the field contamination, we used the Blob subset from TOPCAT for selecting the cluster region in the VPD. Stars are considered members if they lie inside the overdensity cluster region in VPD and have proper motions in RA and DEC within \pm 3 MAD from the mean proper motion; they must also have a clear main sequence in the CMD (Figure 2). These stars must be inside the limiting radius and they should have the same direction of proper motion vectors (Figure 4). The cluster members must have the same angular speed in the space.

To determine the mean proper motion of the cluster, we constructed the histograms and made a Gaussian fitting of the proper motions in both directions RA and DEC, see Figure 3. We found that the mean proper motion of NGC 2158 is $\mathrm{pmRA}=-0.159 \pm 0.165 \mathrm{mas} / \mathrm{yr}$ and $\mathrm{pmDEC}=$ $-2.000 \pm 0.175 \mathrm{mas} / \mathrm{yr}$. These values are in good 
TABLE 1

ESTIMATION OF THE pmRA AND THE pmDEC VALUES OF THE OPEN CLUSTER NGC 2158

\begin{tabular}{cll}
\hline Parameter & \multicolumn{1}{c}{ Values } & \multicolumn{1}{c}{ References } \\
\hline $\operatorname{pm} R A$ (mas/yr) & $-0.159 \pm 0.165$ & Present study \\
& $-0.177 \pm 0.185$ & Cantat-Gaudin et al. (2018) \\
& $+0.002 \pm 0.089$ & Loktin and Popova (2017) \\
& -0.290 & Kharchenko et al. (2013) \\
& +1.430 & Wu et al. (2009) \\
& $-1.220 \pm 0.190$ & Dias et al. (2002) \\
\hline $\operatorname{pm}(\mathrm{mas} / \mathrm{yr})$ & $-2.000 \pm 0.175$ & Present study \\
& $-2.002 \pm 0.173$ & Cantat-Gaudin et al. (2018) \\
& $-0.849 \pm 0.089$ & Loktin and Popova (2017) \\
& -2.480 & Kharchenko et al. (2013) \\
& -3.280 & Wu et al. (2009) \\
& $-3.300 \pm 0.010$ & Dias et al. (2002)
\end{tabular}

agreement with the values obtained by CantatGaudin et al. (2018), see Table 1.

The measured proper motions of the eclipsing binary $[\mathrm{NBN} 2015] 78$ in Gaia DR2 are $\mathrm{pmRA}=-3.252 \pm 0.673 \mathrm{mas} / \mathrm{yr}$ and $\mathrm{pmDEC}=$ $-2.63 \pm 0.596 \mathrm{mas} / \mathrm{yr}$ with relative uncertainties smaller than $20 \%$. The position of the eclipsing binary [NBN2015]78 in VPD is found to be very far away from the cluster region, close to the main sequence of the CMD (Figure 2). It is clearly seen that the direction of the proper motion vector of [NBN2015]78 in Figure 4 is different from the direction of the cluster members. Therefore, in spite of its position in the color magnitude diagrams in the optical and IR (2MASS, Figure 7) this eclipsing binary does not seem to be a member of the cluster.

\subsection{Cluster Center and Radius}

To determine the cluster center we count the stars in right ascension (RA) and declination (DEC). The center of the open cluster NGC 2158 is calculated at the point where the maximum stellar density of the cluster's area is reached, using a Gaussian fitting. The center of the cluster NGC 2158 is found to be at $R A=91.866 \pm 0.077$ and $D E C=24.109 \pm 0.070$, and the corresponduing Galactic coordinates are $\ell=186.629$ and $b=+1.796$ (Figure 5). We find that that the difference between our estimation of the centre of the cluster in $R A$ and $D E C$ and that of Cantat-Gaudin et al. (2018) is $14^{\prime \prime} .4$ and 0'.60, respectively. The difference with the values from Anderson et al. (2013); Wu et al. (2009); Dias et al. (2002) is $43^{\prime \prime} .2$ and $0^{\prime} .72$, respectively (Table 2 ).

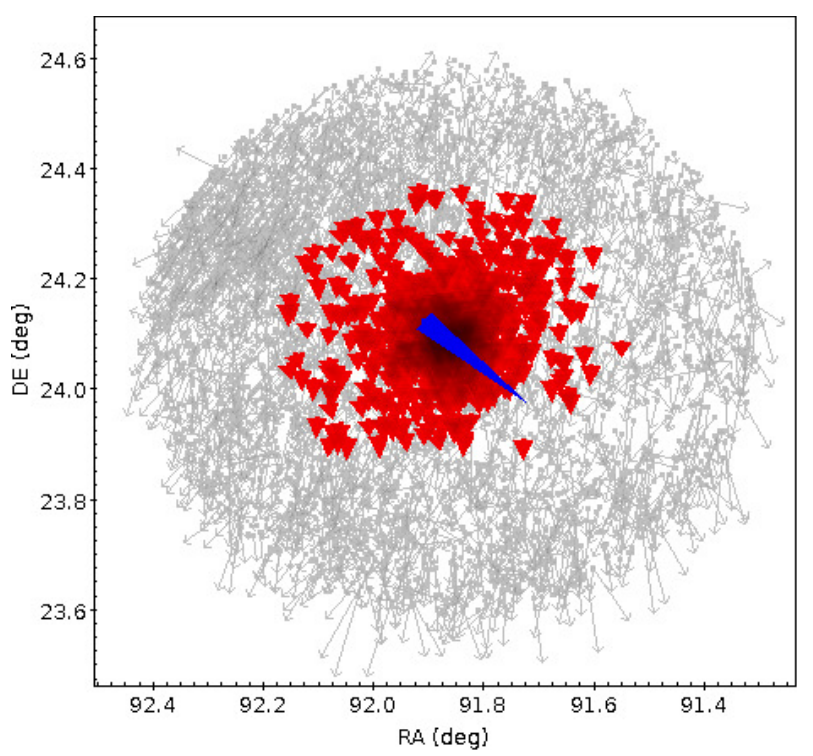

Fig. 4. Projection on the sky of the proper motion vectors. The red vectors (red triangles) represent the cluster members, the blue vector (blue triangle) represents the eclipsing binary [NBN2015]78 and the gray vectors (gray arrows) represent the field stars. The color figure can be viewed online.

To calculate the core and limiting radii, we measure the radial density profile (RDP) of the open cluster NGC 2158, extracting the brightest stars $G \leq 18 \mathrm{mag}$ within a square region of side $2 \mathrm{mas} / \mathrm{yr}$ around the center of the overdensity in the VPD, see Figure 2. We then divide the square area around the cluster into concentric rings. The stars are counted 

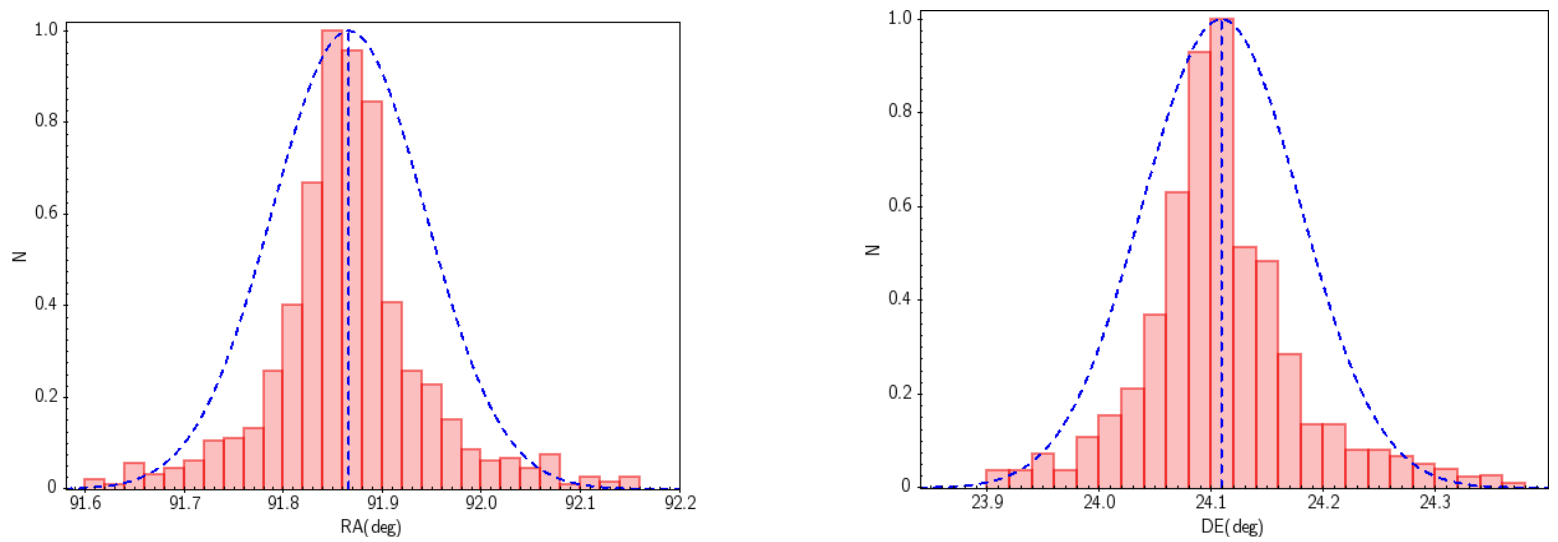

Fig. 5. Inferring the center of NGC 2158 in $R A$ (left panel) and $D E C$ (right panel). The blue lines are Gaussian fits. The color figure can be viewed online.

TABLE 2

ESTIMATIONS OF THE CENTER OF THE OPEN CLUSTER NGC 2158

\begin{tabular}{|c|c|c|}
\hline Parameter & Values & References \\
\hline RA (deg) & $91.866 \pm 0.077$ & Present study \\
\hline \multirow[t]{5}{*}{ RA (hh:mm:ss) } & 06:07:27.84 & Present study \\
\hline & 06:07:26.88 & Cantat-Gaudin et al. (2018) \\
\hline & 06:07:26.40 & Loktin and Popova (2017) \\
\hline & 06:07:25.68 & Kharchenko et al. (2013) \\
\hline & 06:07:25.00 & Anderson et al. (2013), Wu et al. (2009) Dias et al. (2002) \\
\hline DEC (deg) & $24.109 \pm 0.070$ & Present study \\
\hline \multirow[t]{5}{*}{ DEC (dd:mm:ss) } & $24: 06: 32.40$ & Present study \\
\hline & 24:05:56.40 & Cantat-Gaudin et al. (2018) \\
\hline & $24: 05: 52.80$ & Loktin and Popova (2017) \\
\hline & $24: 05: 31.20$ & Kharchenko et al. (2013) \\
\hline & 24:05:48.00 & Anderson et al. (2013), Wu et al. (2009) Dias et al. (2002) \\
\hline \multirow[t]{5}{*}{$\ell(\mathrm{deg})$} & 186.629 & Present study \\
\hline & 186.635 & Cantat-Gaudin et al. (2018) \\
\hline & 186.704 & Loktin and Popova (2017) \\
\hline & 186.635 & Kharchenko et al. (2013) \\
\hline & 186.634 & Anderson et al. (2013), Wu et al. (2009) Dias et al. (2002) \\
\hline \multirow[t]{5}{*}{$\mathrm{b}(\mathrm{deg})$} & 1.796 & Present study \\
\hline & 1.788 & Cantat-Gaudin et al. (2018) \\
\hline & 1.786 & Loktin and Popova (2017) \\
\hline & 1.774 & Kharchenko et al. (2013) \\
\hline & 1.781 & Anderson et al. (2013), Wu et al. (2009) Dias et al. (2002) \\
\hline
\end{tabular}

within each ring and their number divided by the area of each ring. A King (1966) model is then fitted to the radial density profile of NGC 2158 as shown in Figure 6.

$$
\rho(R)=f_{b g}+\left[\frac{f_{0}}{1+\left(R / R_{\text {core }}\right)^{2}}\right],
$$

where $f_{b g}, f_{0}$ and $R_{\text {core }}$ are the background density, the central star density and the core radius of the cluster, respectively. The bestfit values are $f_{0}=67.06 \pm 2.47 \mathrm{stars} / \operatorname{arcmin}^{2}$ and $R_{\text {core }}=1^{\prime} .70 \pm 0$ 0'08 see Table 3 . The limiting radius of NGC 2158 is estimated as $R_{\text {lim }}=17^{\prime} .00$, beyond which stars begin to merge with the background population (see Figure 6, Tadross and Hendy 
TABLE 3

THE RDP PARAMETERS OF THE OPEN CLUSTER NGC 2158

\begin{tabular}{cll}
\hline Parameter & Values & Reference \\
\hline$R_{\text {lim }}(\operatorname{arcmin})$ & 17.00 & Present study \\
& 15.00 & Kharchenko et al. (2013) \\
& 18.60 & Anderson et al. (2013) \\
& 07.50 & Glushkova et al. (2010) \\
\hline$R_{\text {core }}(\operatorname{arcmin})$ & $01.70 \pm 0.08$ & Present study \\
& 02.40 & Kharchenko et al. (2013) \\
& 01.71 & Anderson et al. (2013) \\
\hline$f_{0}\left(\right.$ stars $\left./ \operatorname{arcmin}^{2}\right)$ & 01.00 & Present study \\
\hline
\end{tabular}

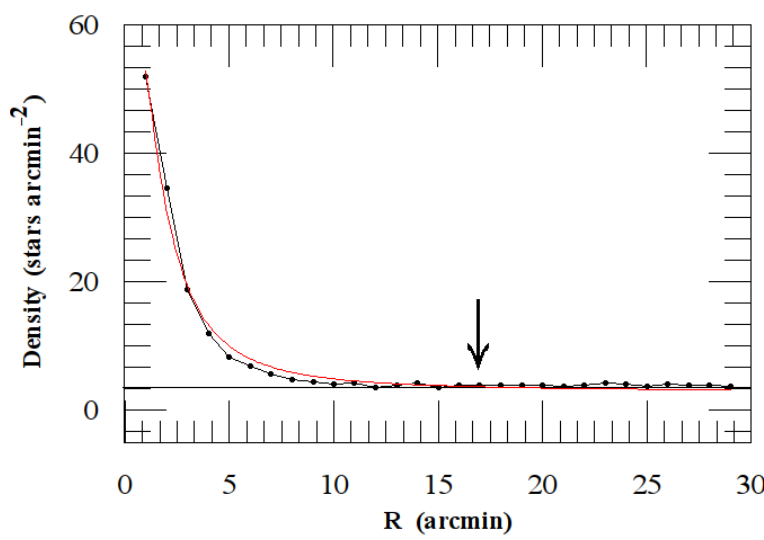

Fig. 6. The radial density profile of NGC 2158. The red curved line is our best-fit King profile). The value of the limiting radius is $R_{\text {lim }}=17^{\prime} .00$. The solid line represents the background density. The color figure can be viewed online.

2016; Hendy 2018). Following Peterson and King (1975), we find that the concentration parameter $C=\log s\left(R_{\text {lim }} / R_{\text {cor }}\right)$ of NGC 2158 is $C=1.00$.

Our estimations of the core and limiting radii are in very good agreement with the ones obtained by Anderson et al. (2013) and Kharchenko et al. (2013), see Table 3, but the limiting radius obtained by Glushkova et al. (2010) is significantly smaller than the one we find.

\subsection{Color Magnitude Diagrams}

To obtain photometric data for multi-color magnitude diagrams (MCMDs), we cross-matched our cluster members in Figure 4 with Gaia, 2MASS and $B V$ data from Nardiello et al. (2015). We obtained 1223 common stars which are members of the cluster. The MCMDs of NGC 2158 are built using optical data from Gaia DR2 $\left[G,\left(G_{B P}-G_{R P}\right)\right]$, Nardiello et al. (2015) $[V,(B-V)]$ and infrared 2MASS data $[J,(J-H)$ and $J-, J-K)]$ are shown in Figure 7.

To obtain the best fit for each CMD, we use the Padova isochrones of Marigo et al. (2017) with a solar metallicity of 0.0152 (Bressan et al. 2013). Using the turn-off point, we find an age of log age $=$ $9.350 \pm 0.050$, that is, and age of $2.240 \pm 0.260$ Gyr. The extinction ratios used for correcting the magnitudes come from the following transformation equations, based on the work by Cardelli et al. (1989) and O'Donnell (1994):

$$
\begin{aligned}
R_{V}=\frac{A_{V}}{E(B-V)} & =3.1, \\
\frac{A_{G}}{A_{V}} & =0.861, \\
\frac{A_{J}}{A_{V}} & =0.296, \\
\frac{A_{K}}{A_{V}} & =0.116,
\end{aligned}
$$

and hence

$$
\begin{aligned}
\frac{E(B-V)}{E\left(G_{B P}-G_{R P}\right)} & =0.785, \\
\frac{E(B-V)}{E(J-H)} & =2.842, \\
\frac{E(B-V)}{E(J-K)} & =1.792 .
\end{aligned}
$$

Table 4 lists the comparison between the fundamental parameters of NGC 2158 obtained here and those obtained in other studies. Using the inferred values of the photometric color excess of NGC 2158, we found that $E\left(G_{B P}-G_{R P}\right)=0.590$, $E(J-H)=0.160$ and $E(J-K)=0.220$. The mean color excess $E(B-V)$ and the intrinsic distance modulus $(m-M)_{0}$ are $0.420 \pm 0.050 \mathrm{mag}$ and 

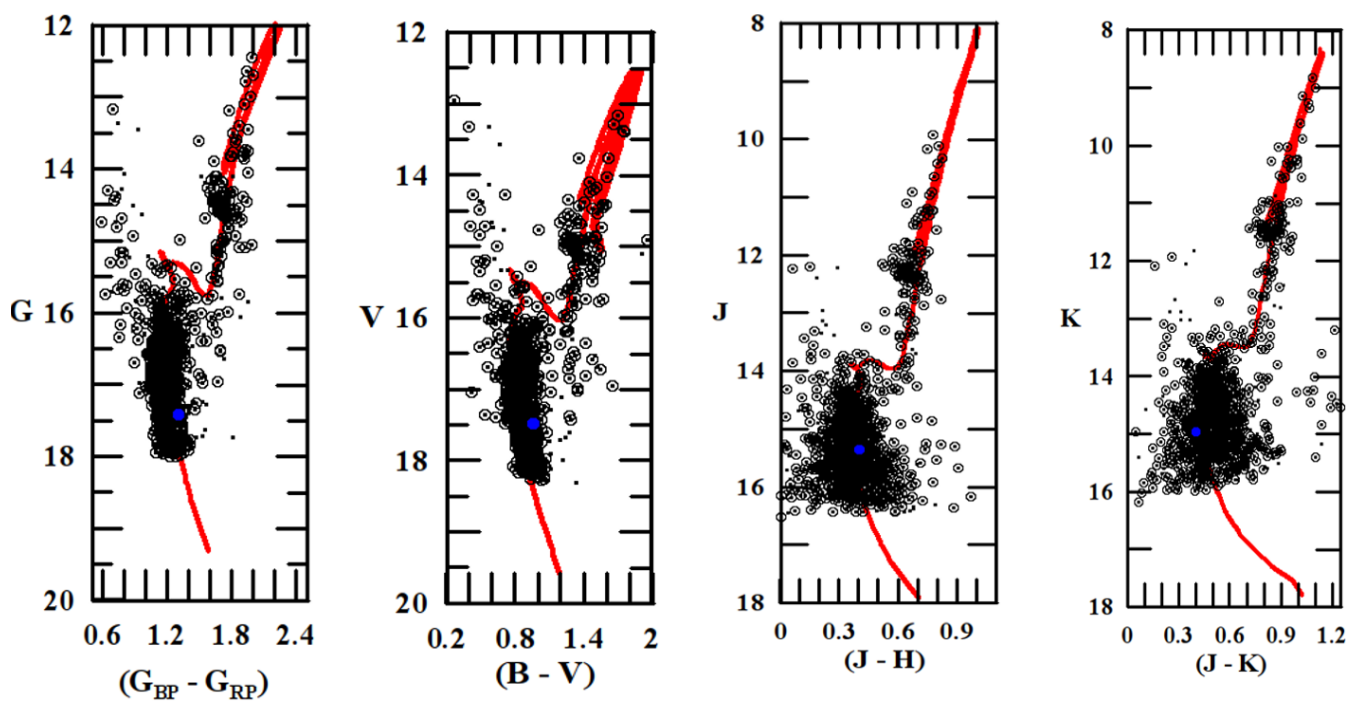

Fig. 7. CMDs of NGC 2158: $\left[G-\left(G_{B P}-G_{R P}\right)\right]$ from Gaia DR2, $[V-(B-V)]$ from Nardiello et al. (2015) and $[J-(J-H) \& J-(J-K)]$ from 2MASS data respectively. The blue circles in the CMDs refer to the position of the eclipsing binary [NBN2015]78. The dots represent 1223 member stars in the optical (Gaia \& $[V-(B-V)]$ ), and the 2MASS data using our criteria. The open circles represent 1031 membesr stars obtained by Cantat-Gaudin et al. (2018) using their UPMASK code to determine membership probabilities. The color figure can be viewed online.

$12.540 \pm 0.130 \mathrm{mag}$ respectively. $E(B-V)$ and $(m-M)_{0}$ are in a good agreement with those estimated by Glushkova et al. (2010), Tadross (2001); Loktin et al. (2001); Dias et al. (2002) and Bedin et al. (2010), see Table 4. The distance of NGC 2158 from the Sun is $d_{\odot}=3224 \pm 200 \mathrm{pc}$, in good agreement with that of Glushkova et al. (2010).

Cantat-Gaudin et al. (2018) calculated the distance of 1229 open clusters by using the UPMASK code of probable cluster members with probabilities larger than 50\%. Lindegren et al. (2018) found that the Gaia DR2 parallaxes are affected by a zero-point offset of -0.029 mas. Cantat-Gaudin et al. (2018) reported in their Table 1 (Vizier database reference $\mathrm{J} / \mathrm{A}+\mathrm{A} / 618 / \mathrm{A} 93)$ the distance and its uncertainties $d, d_{+}$and $d_{-}$as $4535.1,3119.8$ and $8301 \mathrm{pc}$ respectively ${ }^{4}$. We estimate a distance of $d_{\odot}=3224 \pm 200 \mathrm{pc}$ using the best fit of the isochrones in the multi-color magnitude diagrams of optical and 2MASS data (Figure 7), in a good agreement with the smaller value of $d_{+}=3119.8 \mathrm{pc}$ of Cantat-Gaudin et al. (2018).

The published parallax of the eclipsing binary [NBN2015]78 in Gaia DR2 is $\varpi=2.202 \pm 0.409$ mas, a relative uncertainty smaller than $20 \%$. We determine the distance of this binary as $d_{E B}($ Gaia $)=$

\footnotetext{
${ }^{4} d_{+}$and $d_{-}$are the most likely distances when 0.1 mas is added or subtracted to the maximum likelihood parallax.
}

454.2 pc using the inverse of the Gaia parallax. This distance to $[N B N 2015] 78$ is much smaller than the mean distance to NGC $2158 d_{\odot}=3224 \pm 200 \mathrm{pc}$, and therefore [NBN2015]78 is a foreground field star and not a cluster member.

Following Tadross (2011)we assume that the Sun lies at distance $8.2 \mathrm{kpc}$ from the Galactic center, and find, for the following parameters: the distance of NGC 2158 from the Galactic center $\left(R_{g c}\right)$, the projected distances on the Galactic plane from the Sun $\left(X_{\odot}\right.$ and $\left.Y_{\odot}\right)$ and the distance from the Galactic plane $\left(Z_{\odot}\right)$ the values $R_{g c}=11407 \pm 394 \mathrm{pc}$, $X_{\odot}=-3201 \pm 278 \mathrm{pc}, Y_{\odot}=-372 \pm 32 \mathrm{pc}$ and $Z_{\odot}=101 \pm 009 \mathrm{pc}$, respectively. These values for $R_{g c}, X_{\odot}, Y_{\odot}$ and $Z_{\odot}$ are fully consistent with those of Cantat-Gaudin et al. (2018) using $d_{+}=3119.8$ pc, see Table 4.

\section{PHOTOMETRIC STUDY OF THE ECLIPSING BINARY [NBN2015]78 \\ 3.1. Data Analysis}

The photometric analysis of [NBN2015]78 was carried out using the data obtained by Nardiello et al. (2015), using the SBIG STL-11000M camera attached to the Asiago 67/92 cm Schmidt Telescope, available at the Astronomical Observatory of Padova (OAPD-Osservatorio Astronomico di Padova). The 
TABLE 4

FUNDAMENTAL PARAMETERS OF NGC 2158

\begin{tabular}{|c|c|c|}
\hline Parameter & Values & References \\
\hline Solar metallicity & 0.0152 & Present study \\
\hline \multirow[t]{3}{*}{ Members (stars) } & 1223 & Present study \\
\hline & 1380 & Cantat-Gaudin et al. (2018) \\
\hline & 216 & Dias et al. (2002) \\
\hline \multirow[t]{5}{*}{ log age $(y r)$} & $9.350 \pm 0.050$ & Present study \\
\hline & 9.330 & Kharchenko et al. (2013) \\
\hline & $9.300 \pm 0.100$ & Glushkova et al. (2010) \\
\hline & 9.301 & Chen et al. (2003) \& Bedin et al. (2010) \\
\hline & 9.200 & Tadross $(2001)$ \\
\hline \multirow[t]{7}{*}{$E(B-V)$} & $0.420 \pm 0.050$ & Present study \\
\hline & $0.368 \pm 0.021$ & Loktin \& Popova (2017) \\
\hline & 0.333 & Kharchenko et al. (2013) \\
\hline & $0.340 \pm 0.030$ & Glushkova et al. (2010) \\
\hline & 0.420 & Bedin et al. (2010) \\
\hline & 0.360 & Loktin et al. (2001) \& Dias et al. (2002) \\
\hline & 0.400 & Tadross (2001) \\
\hline$E\left(G_{B P}-G_{R P}\right)$ & 0.590 & Present study \\
\hline \multirow[t]{2}{*}{$E(J-H)$} & 0.160 & Present study \\
\hline & 0.107 & Kharchenko et al. (2013) \\
\hline \multirow[t]{2}{*}{$E(J-K)$} & 0.220 & Present study \\
\hline & 0.160 & Kharchenko et al. (2013) \\
\hline \multirow[t]{4}{*}{$(m-M)_{\odot}$} & $12.540 \pm 0.130$ & Present study \\
\hline & $13.545 \pm 0.082$ & Loktin \& Popova (2017) \\
\hline & $12.580 \pm 0.200$ & Glushkova et al. (2010) \\
\hline & 12.980 & Bedin et al. (2010) \\
\hline \multirow[t]{7}{*}{$d_{\odot}(\mathrm{pc})$} & $3224 \pm 200$ & Present study \\
\hline & 3119.800 & Cantat-Gaudin et al. (2018) \\
\hline & 4770 & Kharchenko et al. (2013) \\
\hline & $3300 \pm 300$ & Glushkova et al. (2010) \\
\hline & 5071 & Dias et al. (2002), Wu et al. (2009) \& Gozha et al. (2012) \\
\hline & 3600 & Carraro et al. (2002 ) \& Chen et al. (2003) \\
\hline & 5012 & Tadross (2001) \\
\hline \multirow[t]{3}{*}{$R_{g c}(\mathrm{pc})$} & $11407 \pm 394$ & Present study \\
\hline & 11444 & Cantat-Gaudin et al. (2018) \\
\hline & 13050 & Gozha et al. (2012) \\
\hline \multirow[t]{4}{*}{$X_{\odot}(\mathrm{pc})$} & $-3201 \pm 278$ & Present study \\
\hline & -3097 & Cantat-Gaudin et al. (2018) \\
\hline & -13035 & Wu et al. (2009) \\
\hline & -5035 & Gozha et al. (2012) \\
\hline \multirow[t]{3}{*}{$Y_{\odot}(\mathrm{pc})$} & $-372 \pm 032$ & Present study \\
\hline & -360 & Cantat-Gaudin et al. (2018) \\
\hline & -586 & Wu et al. (2009) \& Gozha et al. (2012) \\
\hline \multirow[t]{3}{*}{$Z_{\odot}(\mathrm{pc})$} & $101 \pm 009$ & Present study \\
\hline & 97.300 & Cantat-Gaudin et al. (2018) \\
\hline & 158 & Wu et al. (2009) \& Gozha et al. (2012) \\
\hline
\end{tabular}



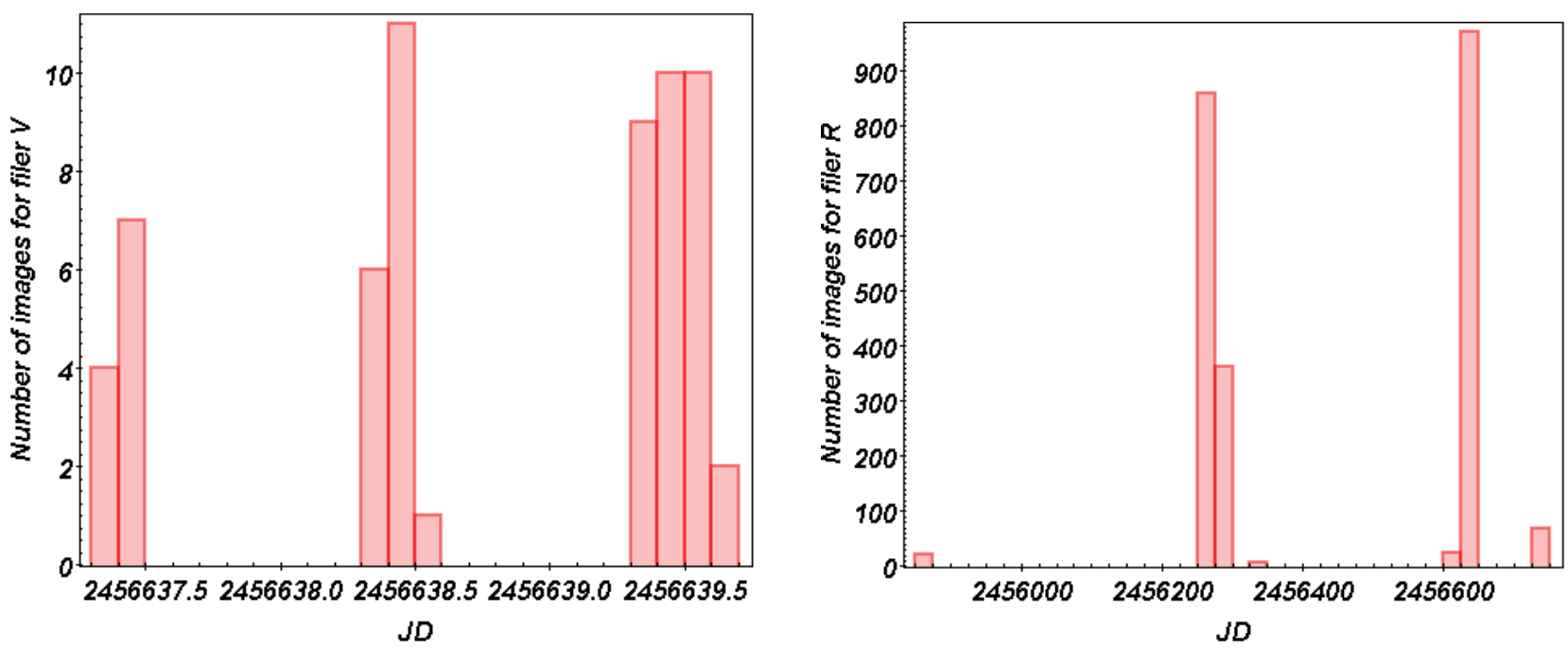

Fig. 8. Histogram of the number of images collected per night during the three campaigns. The histograms refer to observations in the $V$ and $R$ filters. The color figure can be viewed online.

TABLE 5

\section{LOG OF OBSERVATIONS}

\begin{tabular}{ccccc}
\hline Filter & \# Images & $\begin{array}{c}\text { Exp. time } \\
(\mathrm{s})\end{array}$ & $\begin{array}{c}\text { FWHM } \\
(\operatorname{arcsec})\end{array}$ & $\begin{array}{c}\text { Median FWHM } \\
(\operatorname{arcsec})\end{array}$ \\
\hline$V$ & 60 & 180 & $1.24-2.05$ & 1.43 \\
\hline$R$ & 1385 & 15 & $1.35-6.34$ & 2.75 \\
& 27 & 120 & & \\
& 2552 & 180 & & \\
\hline
\end{tabular}

$V, R$ data were collected from a number of images gathered over the three seasons. Table 5 presents the total number of observations in each filter as obtained from Nardiello et al. (2015) and Figure 8 shows the histogram of the observations in each filter.

The period and the times of primary (I) and secondary (II) minima of [NBN2015]78 were obtained for the $V$ and $R$ filters (see Table 6). Using the method of Kwee and Woerden (1956), we infer that the new ephemeris for the system is given by

$\operatorname{HJD}(\operatorname{MinI})=2456639.55464 \pm 0.0015+0.341325^{d} \times E$,

where $E$ is the integer number of cycles. This ephemeris is used to calculate the phases and to draw the light curves in the $V$ and $R$ bands as shown in Figure 9.

\subsection{Light Curve Analysis}

The light curve analysis of the system [NBN2015]78 is performed using the light curves in both $V$ and

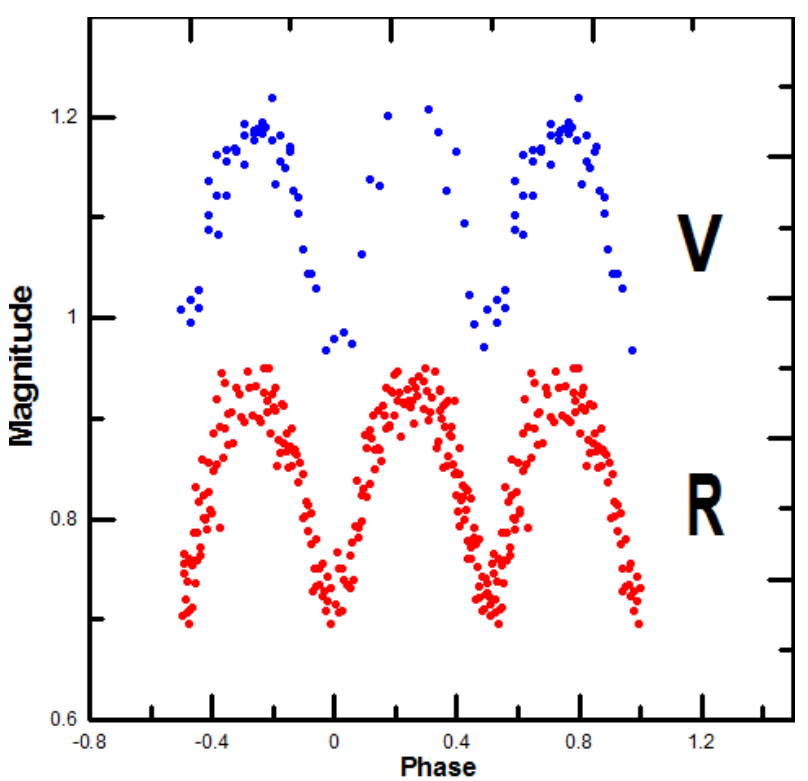

Fig. 9. The observed phase diagram of [NBN2015]78 in the $V$ and $R$ filters. The color figure can be viewed online.

$R$ bands and is carried out using the program of Wilson-Devinney (W-D, Nelson 2009; Wilson and Devinney 1971). We assumed gravity darkening and bolometric albedo exponents appropriate for the convective envelopes of late-type stars $\left(T_{\text {eff }}<7500 \mathrm{~K}\right)$. We adopted $g_{1}=g_{2}=0.32$ (Lucy 1967) and $A_{1}=$ $A_{2}=0.5$ (Rucinski 1969). The limb-darkening coefficients were interpolated from the tables of van 
TABLE 6

\section{TIMES OF PRIMARY AND SECONDARY MINIMA OF [NBN2015]78}

\begin{tabular}{ccc}
\hline Filter & MinI & MinII \\
\hline$V$ & $2456639.555 \pm 0.002$ & $2456638.351 \pm 0.0001$ \\
\hline$R$ & $2456639.547 \pm 0.002$ & $2456639.384 \pm 0.0002$ \\
\hline
\end{tabular}

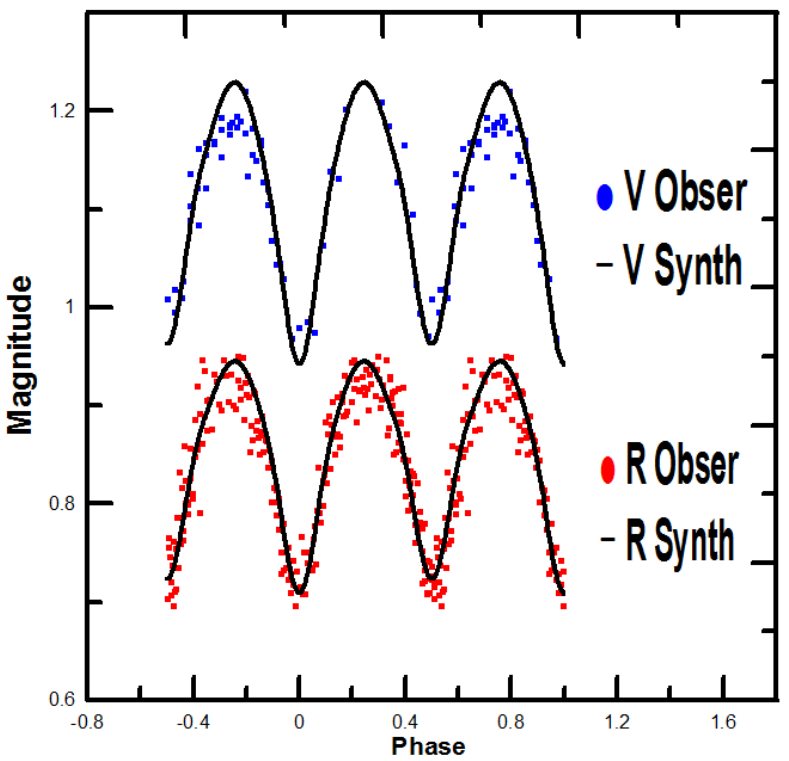

Fig. 10. $V, R$ filters for the system [NBN2015]78 as fitted with the synthetic model (sold line). The color figure can be viewed online.

Hamme (1993) using the logarithmic law and the values $X 1=X 2$ and $Y 1=Y 2$ for the over-contact mode. The effective temperature of the primary star $\left(T_{1}\right)$ is adopted as $4925 \mathrm{~K}$ (Flower 1996), corresponding to a $B-V$ color index of 0.959 from the combined light of both components (Nardiello et al. 2015). The adjusted parameters are the orbital inclination $(i)$, the mean temperature of the secondary star $\left(T_{2}\right)$, the potential of the two components $\Omega=\Omega_{1}=\Omega_{2}$, the mass ratio $(q)$, and the luminosity of the primary star $\left(L_{1}\right)$. We used Mode 3 in the Wilson-Devinney (W-D) program (over-contact mode not in thermal contact). The orbital solutions using both $V$ and $R$ light curves are performed and the accepted parameters are listed in Table 7 . Figure 10 shows the best match between the model and the observed light curves. The solution shows that the primary component is the more massive and the hotter one, with a difference in effective temperature equal to $145 \mathrm{~K}$. According to this solution, the two components of
TABLE 7

THE ORBITAL SOLUTION FOR [NBN2015]78 IN $V$ AND $R$ BANDS

\begin{tabular}{ccc}
\hline Parameter & $V$ & $R$ \\
\hline$\lambda(\AA)$ & 5500 & 7000 \\
$T_{1}(\mathrm{~K})$ & 4925 & 4925 \\
$T_{2}(\mathrm{~K})$ & $4780 \pm 107$ & $4760 \pm 062$ \\
$\mathrm{q}$ & $0.263 \pm 0.035$ & $0.262 \pm 0.010$ \\
$\Omega_{1}=\Omega_{2}$ & $2.354 \pm 0.075$ & $2.374 \pm 0.023$ \\
$g_{1}=g_{2}$ & 0.320 & 0.320 \\
$A_{1}=A_{2}$ & 0.500 & 0.500 \\
$X_{1}=X_{2}$ & $0.643 \pm 0.076$ & $0.643 \pm 0.028$ \\
$Y_{1}=Y_{2}$ & 0.160 & 0.160 \\
$i\left(^{\circ}\right)$ & $69.530 \pm 2.900$ & $67.500 \pm 1$ \\
$\mathrm{r} \mathrm{pole} 1$ & 0.468 & 0.473 \\
$\mathrm{r} \mathrm{side} 1$ & 0.505 & 0.512 \\
$\mathrm{r}$ back 1 & 0.530 & 0.538 \\
$\mathrm{r} \mathrm{pole} 2$ & 0.253 & 0.259 \\
$\mathrm{r}$ side2 & 0.264 & 0.270 \\
$\mathrm{r}$ back2 & 0.297 & 0.308 \\
$L_{1}$ & $0.797 \pm 0.050$ & $0.799 \pm 0.020$ \\
$L_{1}+L_{2}$ & 0.020 & 0.160 \\
\hline${ }^{\circ}(o-c)^{2}$ & &
\end{tabular}

TABLE 8

\section{ABSOLUTE PHYSICAL PARAMETERS FOR [NBN2015]78}

\begin{tabular}{ccccccc}
\hline Element & $M\left(M_{\odot}\right)$ & $R\left(R_{\odot}\right)$ & $T\left(T_{\odot}\right)$ & $L\left(L_{\odot}\right)$ & $M_{\text {bol }}$ & Sp.type \\
\hline Primary & 0.817 & 0.912 & 0.852 & 0.439 & 5.588 & K1 \\
Secondary & 0.214 & 0.858 & 0.827 & 0.345 & 5.849 & K2
\end{tabular}

[NBN2015]78 are of spectral types K1 and K2 (Covey 2007)

Figure 11 shows the geometric configuration of [NBN2015]78 at different phases and the corresponding Roche lobe geometry. The absolute physical parameters of the system are calculated using the empirical relations of Harmanec (1988). The mass of the primary component $M_{1}=0.817 M_{\odot}$, while the mass of the secondary component is directly calculated from the estimated mass ratio of the system $\left(q=M_{2} / M_{1}\right)$ as $M_{2}=0.214 M_{\odot}$. The radii of the two components $R_{1}\left(R_{\odot}\right) ; R_{2}\left(R_{\odot}\right)$ and the bolometric magnitudes $M_{1 b o l}$ and $M_{2 b o l}$ also calculated and listed in Table 8.

\subsection{Evolutionary Status of [NBN2015]78}

Using the calculated physical parameters listed in Table 8 we investigated the current evolutionary sta- 

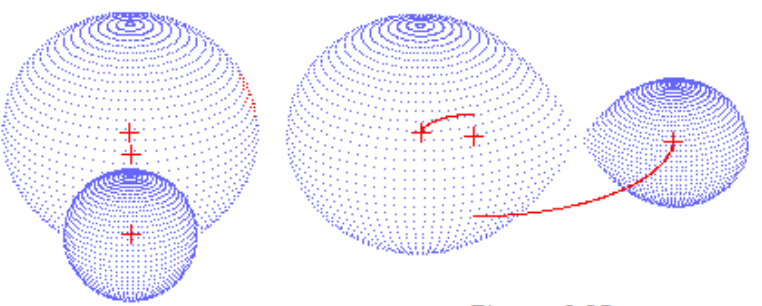

Phase $=0.0$

Phase $=0.25$
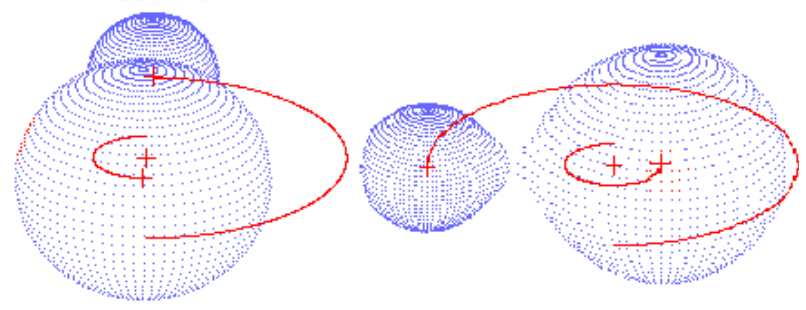

Phase $=0.5$

Phase 0.75

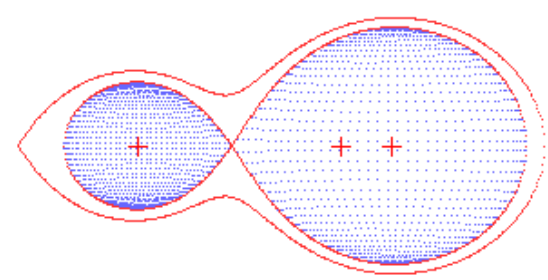

Fig. 11. Geometric configuration at different phases of [NBN2015]78. The color figure can be viewed online.

tus of the system. Figure 12 shows the components of the system in the mass-luminosity $(M-L$, left panel), mass-radius ( $M-R$, middle panel) and masstemperature ( $M-T$, right panel) relations, along with evolutionary tracks computed by Mowlavi et al. (2012) for both zero age main sequence (ZAMS) and terminal age main sequence (TAMS) with solar metallicity $\left(Z_{\odot}=0.014\right)$.

The right panel also shows the location of the components on the empirical $M-T_{\text {eff }}$ relation for intermediate mass stars from Malkov (2007). As it is clear from Figure 12 (left and middle panels) the primary component is lying on the ZAMS track while the secondary component is an evolved star.

\section{DISCUSSION AND CONCLUSIONS}

Using a combination of all the available photometric and astrometric data from the Gaia DR2 and 2MASS catalogues, we presented the optical, nearIR photometric and astrometric properties of the intermediate-age open cluster NGC 2158 and an eclipsing binary system [NBN2015]78 which is found within the same field. The main results of this study can be summarized as follows:

- The cluster membership investigation was performed using a criterion based on the high- precision Gaia proper motions. We found 1223 member stars in Gaia, 2MASS and the BV catalogue of Nardiello et al. (2015), 1031of which being in the catalog of Cantat-Gaudin et al. (2018) with high membership probabilities, above $>60 \%$.

- Using multi-color magnitude diagrams (Gaia DR2, optical BV, 2MASS), we determined the age, colour excess and distance of NGC 2158 as $2.240 \pm 0.260 \mathrm{Gyr}, 0.420 \pm 0.050 \mathrm{mag}$ and $3224 \pm 200$ pc, respectively.

- Using the high-precision Gaia proper motions, we found that the position of the eclipsing binary [NBN2015]78 in VPD is very far away from the cluster region. It is clearly seen that the direction of the proper motion vector of [NBN2015]78 is different from the direction of the cluster members.

- We analyzed the light curves in the $V$ and $R$ bands of the system [NBN2015]78 using the W$\mathrm{D}$ code to obtain the geometric and photometric parameters of the system.

- The [NBN2015]78 system is an over-contact binary with a fill-out factor $=17.5 \%$ and a low mass ratio $q=0.262$.

- The system is classified as being of the A-subtype and the components have spectral types $\mathrm{K} 1$ and $\mathrm{K} 2$.

- The positions of both components of [NBN2015]78 in the mass-radius and massluminosity relations reveal that the primary component is a main sequence star while the secondary is an evolved component.

- The distance to [NBN2015]78 is $454.2 \mathrm{pc}$, while the mean distance to the NGC2158 is equal to $3224 \pm 200$ pc, so we can confirm that the binary system [NBN2015]78 is definitely one of the foreground field stars.

A series of new photometric and spectroscopic observations of [NBN2015]78 is highly recommended for a detailed study of its period stability and to determine more precise physical parameters.

The research is supported by the National Research Institute of Astronomy and Geophysics (NRIAG) and the Science and Technology Development Fund (STDF No. 5217). This work also is supported by the IMHOTEP collaboration program 

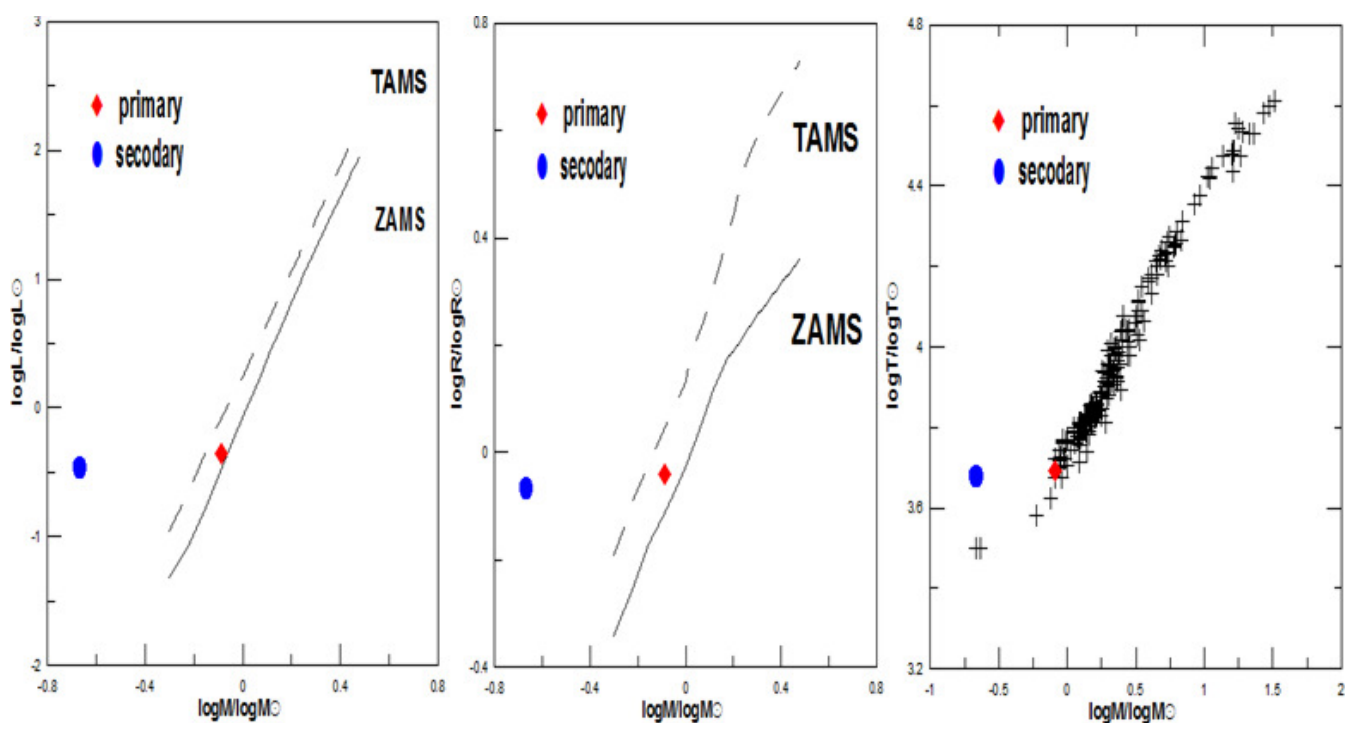

Fig. 12. The position for the components of the system on the mass-luminosity (left), mass-radius (middle) and empirical $M-T_{\text {eff }}$ relation (right) for intermediate mass stars. The color figure can be viewed online.

No. 42088ZK between Egypt and France. We are grateful to the Academy of Scientific Research and to the Service of Scientific Cooperation in both countries for giving us the opportunity to work through this project and providing the necessary support to accomplish our scientific goals. The criticism of the first version of this article by the anonymous referee and the revision of the text done by Prof. David Valls-Gabaud greatly helped us to improve the article and the arguments, and the authors gratefully appreciate this effort. The authors also are very grateful to I. Zead and M. Darwish for helpful discussions. This work has made use of data from the European Space Agency (ESA) mission Gaia https://www.cosmos.esa.int/gaia, processed by the Gaia Data Processing and Analysis Consortium (DPAC, https://www.cosmos.esa. int/web/gaia/dpac/consortium). Funding for the DPAC has been provided by national institutions, in particular the institutions participating in the Gaia Multilateral Agreement. This publication makes use of data products from the Two Micron All Sky Survey, which is a joint project of the University of Massachusetts and the Infrared Processing and Analysis Center/California Institute of Technology, funded by the National Aeronautics and Space Administration and the National Science Foundation. This work has mad use of TOPCAT http://www.starlink.ac. uk/topcat. It has been developed mostly in the UK within various UK and Euro-VO projects (Starlink, AstroGrid, VOTech, AIDA, GAVO, GENIUS,
DPAC) and under PPARC and STFC grants. Its underlying table processing facilities are provided by the related packages STIL and STILTS. This research has made use of Vizier catalogue access tool, CDS, Aladin sky atlas developed at CDS, Strasbourg Observatory, France.

\section{REFERENCES}

Anderson, R. I., Eyer, L., \& Mowlavi, N. 2013, MNRAS, 434, 2238

Bedin, L. R., Salaris, M., King, I. R., et al. 2010, ApJ, 708, 32

Bisht, D., Yadav, R. K. S., \& Ganesh, S. 2019, MNRAS, 482, 1471

Bonnarel, F., Fernique, P., Bienaymé, O., et al. 2000, A\&AS, 143, 33

Bressan, A., Marigo, P., Girardi, L., Nanni, A., \& Rubele, S. 2013, EPJWC, 43, 03001

Cantat-Gaudin, T., Jordi, C., Vallenari, A., et al. 2018, A\&A, 618, 93

Cardelli, J. A., Clayton, G. C., \& Mathis, J. S. 1989, ApJ, 345, 245

Carraro, G., Girardi, L., \& Marigo, P. 2002, MNRAS, $332,705 \mathrm{C}$

Chen, L., Hou, J. L., \& Wang, J. J. 2003, AJ, 125, 1397C

Covey, K. R., Ivezić, Ž., Schlegel, D., et al. 2007, AJ, 134, 2398

Dias, W. S., Alessi, B. S., Moitinho, A., \& Lepine, J. R. D. 2002, A\&A, 389, 871

Ferreira, Filipe A., Santos, J. F. C., Corradi, W. J. B., et al. 2019, MNRAS, 483, 5508F

Flower, P. J. 1996, ApJ, 469, 355 
Gaia Collaboration, Prusti, T., de Bruijne, J. H. J., et al. 2016, A\&A, 595, A1

Gaia Collaboration, Brown, A. G. A., Vallenari, A., et al. 2018, A\&A, 616, A1

Glushkova, E. V., Koposov, S. E., Zolotukhin, I. Yu, et al. 2010, AstL, 36, 75

Gozha, M. L., Borkova, T. V., \& Marsakov, V. A. 2012, AstL, 38, 506

Harmanec, P. 1988, BAICz, 39, 329

Hendy, Y. H. M. 2018, JAsGe, 7, 180H

Kharchenko, N. V., Piskunov, A. E., Schilbach, E., et al. 2013, A\&A, 558, 53

King, I. R. 1966, AJ, 71, 64

Kwee, K. \& Van Worden, H. 1956, BAN, 12, 327

Lindegren, L., Hernandez, J., Bombrun, A., et al. 2018, A\&A, 616, A2

Loktin, A. V., Gerasimenko, T. P., \& Malysheva, L. K. 2001, A\&AT, 20, 607

Loktin, A. V. \& Popova, M. E. 2017, AstBu, 72, 257

Lucy, L. 1967, ZA, 65, 89

Lynga, G. 1987, The Open Star Clusters Catalogue, 5th edition: (http://cdsarc.u-strasbg.fr/viz-bin/VizieR? -meta.foot\&-source=VII/92A)

Malkov, O. Yu. 2007, MNRAS, 382, 1073
Marigo, P., Girardi, L., Bressan, A., et al. 2017, ApJ, 835, 77

Mowlavi, N., Eggenberger, P., Meynet, G., et al. 2012, A\&A, 541, 41

Nardiello, D., Bedin, L. R., Nascimbeni, V., et al. 2015, MNRAS, 447, 3536

Nelson, R. H. 2009, WDwint56a, available from: (https: //www.variablestarssouth.org/bob-nelson)

O'Donnell, J. E. 1994, ApJ, 422, 158

Peterson, C. J. \& King, I. R. 1975, AJ, 80, 427

Ruciński, S. M. 1969, AcA, 19, 125

Saad, M. S., Darwish, M. S., Nasser, M. A., et al. 2016, NewA, 47, 24S

Skrutskie, M. F., Cutri, R. M., Stiening, R., et al. 2006, AJ, 131, 1163

Stassun, K. G. \& Torres G. 2018, ApJ, 862, 61S

Tadross, A. L. 2001, NewA, 6, 293 . 2011, JKAS, 44, 1

Tadross, A. L. \& Hendy, Y. H. M. 2016, JKAS, 49, 53T

Taylor, M. B. 2005, ASPC, 347, 29

Van Hamme, W. 1993, AJ, 106, 2096

Wilson, R. E. \& Devinney, E. J. 1971, ApJ, 166, 605

Wu, Z-Y., Zhou, X., Ma, J., \& Du, C-H. 2009, MNRAS, 399,2146

A. E. Abdelaziz, Y. H. M. Hendy, S. M. Saad, and A. Shokry: Astronomy Department, National Research Institute of Astronomy and Geophysics (NRIAG), Helwan, Cairo, Egypt (abdelazizeid3, y.h.m.hendy@gmail.com).

K. A. Edris and F. Y. Kamal: Department of Astronomy and Meteorology, Faculty of Science, Al-Azhar University, Cairo, Egypt.

S. M. Saad: Kottamia Center of Scientificc Excellence for Astronomy and Space Sciences (KCScE), STDF No. 5217. 